\title{
Template assisted synthetic method for preparation of arrays of metallic nanowires
}

\author{
Miracle Warjri and Jyoti Narayan* \\ Department of Basic Sciences and Social Sciences, School of Technology \\ North Eastern Hill University, Shillong-793022, Meghalaya, India. \\ *Email: jnarayan.nehu@gmail.com
}

Nanowires are defined as structures with diameter confined to the order of a nanometer $\left(10^{-9}\right.$ meters $)$. They may be regarded to possess a lateral size constrained to tens of nanometers or less and an unconstrained longitudinal size (one dimensional). They can be semiconducting, superconducting, metallic or insulating materials. Nanowires can be synthesized using electrochemical deposition, electrophoretic deposition, vapor deposition, template filling via capillary force, vapor-liquidsolid (VLS) method, chemical conversion and centrifugation. Template assisted synthesis method has been favorably used for the preparation of metallic nanowires using various templates like, anodized alumina membrane (AAM) [1], radiation track-itched polycarbonate membrane (RTPM) [2] and indium tin oxide membrane (ITOM) [3] through electrochemical deposition technique (bottom-up). Systematic uniform growth of nanowires within limited time can be obtained. Keeping in view, array of metallic nanowires/nanorods/nanofibres $(\mathrm{Cu} / \mathrm{CuO}, \mathrm{Ni} / \mathrm{NiO})$, on different templates, varying duration of deposition, using three-electrode system at room temperature have been synthesized by potentiostatic electrochemical metal deposition (EMD) technique (Figure 1 and Figure 2). Characterization of the various templates as well as the synthesized nanowires was done using atomic force microscopy (AFM), UV-visible spectroscopy, high resolution transmission electron microscopy (HRTEM), EDAX and scanning electron microscopy (SEM). The voltammetry and the phenomenon of reduction of the charged growth species at the growth or deposition surface of the synthesized nanowires using $\mathrm{CH}$-analyzer at variable external electric field was studied. The results revealed uniform longitudinal growth and the lateral size constrained to nanometer diameter of the nanowires. Since nanowires have unique optical, magnetic and electrical properties, because of their shape, size and reduced dimensionality, variable applications such as sensors, photocatalysts, semiconducting and energy storage devices resulted.
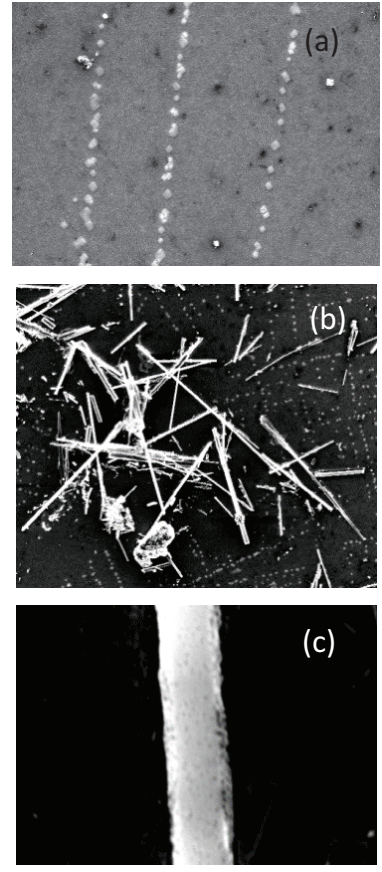

Figure 1: SEM images of $\mathrm{Cu}$ nanowires deposited on ITO template; (a) seedling of nanoparticles, (b) nanowires grown and (c) Single nanowire

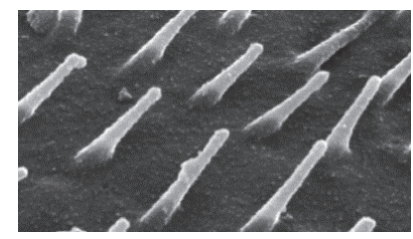

Figure 2: SEM image of $\mathrm{Cu}$ nanowires grown on Polycarbonate membrane

\section{References}

1. R. Kaur, International Journal on Organic Electronics 3 (2014).

2. L.A. Meiera, A.E. Alvareza, S.G. Garcíaa, M.C. del Barrioa, Procedia Materials Science 8 (2015) 617.

3. J. Narayan, S. Choudhary, International Scholarly and Scientific Research \& Innovation 6(7) (2012) 76. 